\title{
Neonatal Tachyarrhythmia, AE
}

National Cancer Institute

\section{Source}

National Cancer Institute. Neonatal Tachyarrhythmia, AE. NCI Thesaurus. Code C154948.

An adverse event in a newborn characterized by non-sinus rhythm with an abnormally high heart rate for age. 